\title{
Demokratie in Venezuela: Präsidentialismus, Parteien und Wahlen
}

\author{
Von Wolfgang Spoerr
}

\section{Einleitung}

In der neueren politikwissenschaftlichen Diskussion sind institutionelle Rahmenbedingungen wieder stärker in den Mittelpunkt des Interesses gerückt, nachdem lange Zeit vorwiegend ökonomische Erklärungsansätze Beachtung fanden. Inzwischen besteht eine breite Bereitschaft, auch Variablen wie Wahlrecht beziehungsweise Wahlsystem, Parteiensystem und Präsidentialismus/Parlamentarismus in die Diskussion einzubeziehen, während in den 70er Jahren die Tendenz bestand, derartige Fragestellungen als unerheblich oder gar von den wirklichen Problemen ablenkend abzuqualifizieren. 1 Insbesondere der Einfluß des jeweiligen Parteiensystems und der Kontrast Parlamentarismus/Präsidentialismus werden diskutiert.2 Dabei kann die Diskussion anknüpfen an die Ergebnisse der historischen Forschung zum Komplex des Zusammenbruches der Demokratien in Europa in den 20er Jahren, auch wenn eine Übertragung aufgrund der völlig anderen kulturellen, historischen und ökonomischen Umstände im heutigen Lateinamerika kaum in Frage kommt und wissenschaftlich wenig ergiebig erscheint. Mit mehr Berechtigung ist versucht worden, die Transitionsprozesse Lateinamerikas und Südeuropas vergleichend gegenüberzustellen. 3

1 vgl. nur Evers, Tilman Tönnies: Demokratie ohne Demokraten - Politische Entwicklung und Wahlrecht in Argentinien, VRÜ 1972, S.117-143, S.117f.

2 siehe etwa: Linz, Juán J.: Democracy - Presidential or Parliamentary: Does it Make a Difference?, Paper prepared for the 83rd Annual Meeting of the American Political Science Association, Chicago 1987; Nohlen, Dieter: Presidencialismo, sistema electoral y sistema de partidos políticos en America Latina. Ponencia presentada en el I Curso Anual Interamericano de Elecciones de CAPEL, Sán José (Costa Rica) 1987; Nohlen,Dieter/Solari, Aldo: Reforma Política y consolidación democrática en Europa y America Latina, Caracas 1988, Kaplan, Marcos: Partidos Políticos, Estatismo y Presidencialismo en la América Latina contemporanea, Cuardernos de CAPEL 1, San José (Costa Rica) 1987, zurückhaltend De Riz, Liliana: Régimen de gobiemo y gobemabilidad Parlamentarismo en Argentina?, in Nohlen/Solari, a.a.O., S.273-285; für Parlamentarisierung Pérez, Romeo, El parlamentarismo. La reforma necesaria para Uruguay, in Nohlen/Solari, a.a.O., S.287-300; Fernández Baeza, Mario: Sistema parlamentario en Chile. Entre la razón y la tradición, in Nohlen/Solari, a.a.O., S.301ff.

3 etwa von O'Donnell, Guillermo/Schmitter, Philippe C./Whitehead, Lawrence (Hg.): Transitions from Authoritarian Rule - Prospects for Democracy. Baltimore/London 1986. 
Im Gegensatz zu Spanien, Portugal und Griechenland lautet der allgemeine Befund für Lateinamerika, daß es dort nicht gelungen ist, demokratische Systeme dauerhaft zu stabilisieren. Vielmehr sind die Staaten südlich des Rio Grande von einer zyklischen Entwicklung, 4 einem Hin- und Herpendeln zwischen autoritärer und konstitutioneller Herrschaft geprägt. Auch angesichts der nicht zu leugnenden demokratischen Tendenz der 80er Jahre (nur noch Chile, Paraguay, Kuba und Nicaragua waren 1988 diktatorisch regiert), ist es für Euphorie noch viel zu früh. Schon öfter gab es in Lateinamerika Wellen der Demokratisierung; und ebenso ist nicht zu verkennen, daß demokratische Systeme derzeit schon wieder gefährdet sind (Ecuador) bzw. zur scheindemokratischen Fassade für autoritäre "hombres fuertes" werden konnten (Panama).

Gerade aus dieser Skepsis bezieht die Fragestellung nach den Stabilitätsbedingungen für demokratische Systeme ihre bleibende Aktualität. Im Gegensatz zu zahlreichen Untersuchungen, die die Thematik negativ angehen, also Zusammenbrüche konstitutioneller Regime analysieren, soll hier der umgekehrte Weg eingeschlagen werden und der lateinamerikanische "deviant case" Venezuela daraufhin untersucht werden, inwiefern institutionelle Rahmenbedingungen dort eine Rolle spielen beim Gelingen einer demokratischen Erfahrung.

Die venezolanische Demokratie wurde stets von Unkenrufen begleitet. So wurde etwa 1973 ein Militärputsch prophezeit.5 Seither sind 16 Jahre vergangen. Trotz einer skeptischen Einstellung der Bevölkerung und Wissenschaft zu einzelnen Aspekten des politischen Systems, auf die häufig verwiesen wird, 6 die aber durch den bestehenden festen Grundkonsens über demokratische Spielregeln zu relativieren ist, ist die venezolanische Demokratie stabiler als jede andere südamerikanische. Seit 1958, also seit über 30 Jahren, wird Venezuela demokratisch regiert, seit 25 Jahren wechseln sich die beiden großen Parteien regelmäßig in der Regierung ab. 1988 gelang es nun einer Partei erstmals wieder, eine zweite Amtsperiode zu erzielen. Akzeptiert man, daß politische Instabilität der lateinamerikanische Normalfall ist, so ist Venezuela der eigentlich erklärungsbedürftige Fall.

4 Nohlen, Dieter: Mehr Demokratie in der Dritten Welt? Über Demokratisierung und Konsolidierung der Demokratie in vergleichender Perspektive, APuZ 1988, S.3-18, S.3, erweiterter Nachdruck in spanisch, Más Democracia en America Latina. Democratización y consolidación de la democracia en una perspectiva comparada. Estudios Sociales (Santiago de Chile), 1989, S.9-43; Mols, Man fred: Demokratie in Lateinamerika, Stuttgart 1985, S.14.

5 etwa von Lindenberg, Klaus: Venezuela vor den Wahlen 1973, unveröffentlichtes Manuskript, Friedrich-Ebert-Stiftung/ILDIS, Caracas 1973, S.22.

6 vgl. etwa: Baloyra, Enrique A.: Public Attitudes Toward the Democratic Regime, in Martz, John D.IMyers, David: Venezuela - The Democratic Experience, New York 1977, S.47-63, S.49f; Boeckh, Andreas: Venezuela, in: Nohlen, Dieter/Nuscheler, Franz (Hg.): Handbuch der Dritten Welt, Bd.2, 2.Auflage 1982; Rey, Juán Carlos, El futuro de la democracia en Venezuela, in Silva Michalena, José A. (Hg.): Venezuela hacia el 2000, Caracas 1988, S.183-245, S.188., nachgedruckt in: Sintesis 5: Venezuela, Madrid 1988, S.171-220. 


\section{Die Vorgeschichte der Demokratie: Die belastete Tradition in Venezuela}

Der demokratische Erfolg Venezuelas wird umso erstaunlicher, wenn man sich vor Augen führt, daß das Land - selbst im lateinamerikanischen Vergleich - eine ausgesprochen belastete Vorgeschichte aufweist. 7 Die Zeit vor 1958 war von langjährigen Diktaturen geprägt; lediglich von 1945-48 bestand ein kurzes demokratisches Zwischenspiel. Unter der Hegemonie der späteren demokratietragenden Partei AD8 wurde ein Populismus praktiziert, wie er in vielen Ländern Lateinamerikas zur historischen Belastung der Demokratien werden sollte. Exzesse, unter anderem gegen die Kirche, trugen zum baldigen Scheitem des, wie es in der venezolanischen Geschichtsschreibung heißt, "trienio populista" bei.

Die für instabile Demokratien so charakteristische Bereitschaft der an sich demokratischen Parteien, antisystemisch zu arbeiten, war auch in Venezuela weit verbreitet. Noch der letzte Putsch des Diktators Pérez Jimenez 1948 fand - angesichts der Performanz der AD-Regierungen nicht ganz unverständlich - mit Billigung aus anderen demokratischen Parteien statt.9 Die Herausbildung demokratischer Institutionen war in Venezuela besonders schwach, wogegen ein starkes Militär, das bereits Simon Bolívar von Venezuela als der "Kaseme Südamerikas" sprechen ließ,10 auf eine ungebrochene Tradition von Interventionen in den politischen Prozeß zurückblickte.11 Noch das erste Jahrzehnt der Demokratie war von einem Guerilla-Krieg geprägt, der in seiner Intensität den bewaffneten Auseinandersetzungen in vielen anderen Staaten Lateinamerikas nicht nachstand. Zu Beginn der 60er Jahre kam es zu mehreren Staatsstreichversuchen.

\section{Herkömmliche Erklärungsansätze für den venezolanischen Erfolg}

Ein Teil der Literatur verweist zur Erklärung der venezolanischen Stabilität auf eine offenkundige Besonderheit Venezuelas im lateinamerikanischen Kontext: Der gewaltige Rohölreichtum, der dem Land einen ständigen Strom von Deviseneinnahmen beschert, ohne daß es befürchten müßte, daß die Quelle bzw. die Reserven in absehbarer Zeit versiegen werden. Das OPEC-Gründungsmitglied Venezuela lebt im wesentlichen von der Ausbeutung seines natürlichen Ressourcenreichtums. Die Abhängigkeit seiner Volkswirtschaft von den Öleinnahmen besteht nicht nur direkt im petrochemischen Sektor, sondern auch in Teilen der Volkswirtschaft, die mit der Olförderung bzw. Produktion direkt gar nichts zu

7 Historische Belastungen der Lateinamerikanischen Demokratien betont Mols, a.a.O. (Fn.4), S.51ff.

8 Alianza Democrática, siehe im einzelnen dazu unten.

9 vgl. dazu im einzelnen Werz, Nikolaus: Parteien, Staat und Entwicklung in Venezuela. München/ Köln/London 1983, S.56f.

10 vgl. Werz, a.a.O. (Fn.9), S.18ff.

11 Die Einbindung des Militärs in das demokratische System wird dargestellt bei Piwonka, Felipe Agüero, Militares y Democracia en Venezuela, Sintesis 5: Venezuela, Madrid 1988, S.297-315. 
tun haben. Die vorliegenden Daten zeigen sehr eindrucksvoll, wie das Konsum- und Investitionsverhalten der venezolanischen Wirtschaftssubjekte, auch solcher, die nicht direkt vom Ölsektor abhängen, von den tatsächlichen und erwarteten Rohöleinnahmen abhängt.12

Auf das politische System wirkt sich der Ressourcenreichtum aus, indem er einen höheren Wohlstand, das "Wegsubventionieren"13 ökonomischer und politischer Konflikte und schließlich die für Venezuela typische breite Kooptations- und Verbeamtungsstrategie auch gegenüber kritischen Kreisen ermöglicht. Aber auch materielle Fortschritte sind in Venezuela nicht zu übersehen. Caracas ist in wenigen Jahrzehnten zur modernsten Stadt Spanisch-Amerikas geworden. Soziale Indikatoren wie etwa die Lebenserwartung haben sich erheblich verbessert.14 Von 1960 bis 1980 stieg die Lebenserwartung von 59 auf 67 Jahre, sank die Kindersterblichkeit von 12 auf 5 pro 1000 Kinder von 1 bis 4 Jahren und stieg die Alphabetisierungsrate von 63 auf 82 Prozent. 15

Dennoch ist gegenüber der Überbetonung ökonomischer Determinanten des venezolanischen politischen Systems Skepsis angebracht: In Relation gesetzt zur natürlichen Ressourcenausstattung des Landes ist die ökonomische und soziale Performanz des Landes traurig. Die realen Wohlstandsgewinne breiter Bevölkerungsschichten durch die oft gelobte sozialreformerische Politik der venezolanischen Regierungen gehen nicht wesentlich über die von den eher traditionell-oligarchischen kolumbianischen Parteien erreichten hinaus. 16 Auch Venezuela zeichnet sich durch ein hohes $\mathrm{Ma}$ an struktureller Heterogenität17 aus. Staatliche Eingriffe in den Wirtschaftsprozeß bewirken ineffiziente Marktverzerrungen in hohem Ausmaß, künstlich niedrig gehaltene Nahrungsmittelpreise begünstigen die städtische, nahrungsmittel-konsumierende Bevölkerung auf Kosten der ländlichen, produzierenden und führen dazu, daß Venezuela trotz seiner günstigen Möglichkeiten immer noch Nahrungsmittelimporteur ist (zeitweise mußte der Tropenstaat sogar Bananenblätter einführen), obwohl Venezuela seine 17-Millionen-Bevölkerung leicht selbst emähren könnte und $\mathrm{da} \beta$ die Landflucht trotz aller Gegensteuerungsversuche des politisch-administrativen Systems anhält. Ökonomisch zumindest zweifelhafte, wenn nicht sogar sinnlose Großpro-

12 Dies wird überzeugend empirisch nachgewiesen von Vaez-Zadeh, Reza: Oil Wealth and Economic Behaviour: The Case of Venezuela, 1965-81, unveröffentlichtes Manuskript, IMF Working Paper 88/56 vom 30.Juni 1977.

13 Boeckh, a.a.O. (Fn.6), S.382.

14 vgl. die optimistische Uminterpretation der lateinamerikanischen Entwicklung bei Hirschman, Albert O.: The Political Economy of Latin American Development - Seven Exercises in Retrospection, LARR XXII-3 (1987), S.7-36.

15 vgl. Nohlen/Nuscheler, a.a.O. (Fn.6), Bd.1, S.492.

16 vgl. zur Unterscheidung Reform-/Statuspartei und zur Gegenüberstellung Venezuela/Kolumbien in diesem Kontext Mols, a.a.O. (Fn.4), S.141ff.

17 vgl. zu diesem Konzept aus einer Fülle von Literatur die zusammenfassende Darstellung von Nohlen, Dieter/Sturm, Roland: Über das Konzept der strukturellen Heterogenität, a.a.O. (Fn.6), Bd.1, S.92-116. 
jekte saugen zur Befriedigung des nationalen Prestiges von Teilen der Elite Schwämmen gleich die öl- und kreditfinanzierten Devisen- und Kapitalzufuhren auf. In allen sozialen Schichten herrscht eine import- statt produktionsorientierte Kompradoren-Haltung vor, die in Verbindung mit einer lange Zeit künstlich wahnwitzig hochgehaltenen nationalen Währung (was ebenfalls mit Öleinnahmen finanziert wurde) dafür sorgte, daß Venezolaner in den Einkaufsparadiesen Floridas durch ihre sprichwörtliche Formulierung "dame dos"18 bekannt wurden. Im ökonomischen und sozialen Bereich sind die Ergebnisse - auch absolut, nicht nur relativ zur Ressourcenaussattung - mangelhaft.19 $\mathrm{Zu}$ welchen Folgen die Unzufriedenheit der Venezolaner mit ihrer wirtschaftlichen Situation führen, zeigten die Unruhen im Februar 1989.

Den Venezolanem konnte das aber schon vorher nicht verborgen geblieben sein. Wenn es im Gegensatz zur ökonomischen und sozialen Performanz in politischer Hinsicht zu Erfolgen kam, so kann dies wohl kaum durch die wirtschaftlichen Mißerfolge ausreichend erklärt werden, vor allem nicht, seit Venezuela mit der Bolívar-Abwertung 1983 und dem Ölpreisverfall den Gürtel erheblich enger schnallen muß und weiten Bevölkerungsteilen in den letzten Monaten der Regierung Herrera Campins und während der Amtsperiode von Präsident Lusinchi drastische Einschränkungen auferlegt wurden. Eine auf die exteme, ökonomische Variable Ölrente zurückgreifende Erklärung greift zu kurz; wie überhaupt Versuche, politische Entwicklung negativ oder positiv mit ökonomischer Entwicklung zu korrelieren, gerade in Lateinamerika an den historischen Tatsachen scheitem - sei es der modernisierungstheoretische Ansatz, wonach mit ökonomischer und sozialer Entwicklung demokratische Reife entsteht (Gegenbeispiel die Neuen Militärregime des Cono Sur), sei es der Versuch O'Donnells20, die Autoritarismen positiv als Folge sich vertiefender Industrialisierung darzustellen. Auch das interne, sozioökonomische Argument, die politische Stabilität Venezuelas beruhe darauf, daß Eliten sich darauf einigten, keine sozialen Reformen vorzunehmen,21 verfängt nicht. Schließlich ist Venezuela geradezu ein Paradebeispiel dafür, wie reformistische Kräfte eine Vielzahl unterschiedlicher Reformen fordem und auch durchführen22, ohne damit dauerhafte und uneingeschränkte Erfolge zu erzielen. Möglicherweise ist die richtige Konsequenz daraus, genauer nach den Erfolgsaussichten zu fragen, die gutgemeinte, oft pauschal als sozial oder fortschrittlich empfohlene, aber selten kritisch hinterfragte Eingriffe bieten.

18 Spanisch in etwa für "Geben Sie mir zwei davon".

19 Ein optimistischeres Bild auch der ökonomischen Entwicklung Venezuelas zeichnet Abente, Diego: Venezuelan Democracy Revisited. LARR 1987, S.225-240.

20 O'Donnell, Guillermo, Modemization and Bureaucratic Authoritarianism, Berkeley 1973, passim.

21 in diesem Sinne O'Donnell, Guillermo: Introduction totheLatinAmerican Cases, in: O'Donnell/Whitehead/Schmitter (Hg.), a.a.O. (Fn.3), S.9.

22 etwa zur Arbeitsgesetzgebung und der damit verbundenen Begünstigung, aber auch Kooptation der Gewerkschaften McCloy, Jennifer L., Labor and the State in a Party-Mediated Democracy - Institutional Change in Venezuela, 1989 LARR 35-67, insbes. S.54-61. 
Auch die einzelnen Argumente, die etwa Terry Lynn Karl ${ }^{23}$ zur Stärkung ihrer These von der von Oleinnahmen stabilisierten Demokratie anführt, sind nicht alle korrekt. Zwar ist richtig, daß die Olrente vorwiegend dem urbanen Venezuela zugute kam. Falsch ist jedoch, $\mathrm{da} ß$ gerade das "urban Venezuela provided a fertile ground for a reformist regime" 24. Vielmehr hatten die venezolanischen Reformparteien, insbesondere die $A D$, in den Großstädten ihre größte Schwäche. Die Massenbasis der Alianza Democratica (AD) lag lange Zeit fast ausschließlich auf dem Land; ähnliches gilt - mit der regionalen Asymmetrie im Westen des Landes - auch für die COPEI. Bis Ende der 60er Jahre bildete die großstädtische Bevölkerung Caracas die wichtigste Basis für Kandidaten am Rande oder außerhalb des demokratischen Spektrums mit ausgeprägt antisystemischem Diskurs. Bei den Unruhen in den großstädtischen Slums im Februar 1989, die von den völlig unvorbereiteten Sicherheitskräften unter großem Blutvergießen bekämpft wurden, zeigte sich die latente Instabilität der urbanen Bevölkerung erneut.

Auf politisch-elitentheoretischer Basis ist Venezuela als Beispiel dafür genannt worden, wie demokratische Stabilisierung auf der Basis von politischen (und auch ökonomischen) "pacts" erfolgt. 25

In Venezuela wird die These auch dadurch genährt, daß für die unmittelbare Úbergangszeit ausdrückliche Verhandlungen und Übereinkünfte zwischen den demokratischen Spitzenpolitikern historische Tatsachen sind. Im Punto-Fijo-Abkommen einigten sich die Spitzenvertreter der systemtragenden Parteien $A D$, COPEI und URD unter anderem auf die Konsolidierung demokratischer Herrschaftsformen und eine gemeinsame Verteidigung der konstitutionellen Regierung im Falle eines Staatsstreiches.26 Das Konzept der Vertreter der Idee von "political pacts" ist jedoch weitergehend. In Venezuela nach 1965 und in vielen anderen lateinamerikanischen Staaten können damit nur "stillschweigende Pakte"27 gemeint werden. So erweitert besagt das Konzept nicht mehr als die Selbstverständlichkeit, $\mathrm{da}$ die Bereitschaft der Eliten, das System nicht zu bekämpfen, mithin ein Grundkonsens über elementare Spielregeln des politischen Prozesses, Stabilitätsvoraussetzung politischer Systeme ist. Diese Binsenweisheit (auch in Großbritannien und den USA könnte man einen "stillschweigenden Pakt" über die Spielregeln des politischen Prozesses diagnostizieren) beschreibt nur, sie erklärt nichts.

23 Karl, Terry Lynn: Petroleum and Political Pacts: The Transition of Democracy in Venezuela, in O'Donnell/Schmitter/Whitehead, a.a.O. (Fn.3), S.196-236.

$24 \mathrm{Karl}$, a.a.O. (Fn.18), S.201.

25 ebenfalls von $\mathrm{Karl}$, a.a.O. (Fn.18).

26 Das "punto-fijo"-Abkommen ist abgedruckt in Sintesis 5: Venezuela, Madrid 1988. S. .

27 in diesem Sinne ausdrücklich $O^{\prime}$ Donnell, der das Fehlen ausdrücklicher ("explicit") Pakte anerkennt, a.a.O. (Fn.3), S.12ff. 
Weite Teile insbesondere der US-amerikanischen Literatur erklären die venezolanische Situation personalistisch mit historisch-kontingenten, nicht übertragbaren Erfahrungen. Stark betont wird dabei die Rolle, die fähige, anerkannte und von demokratischen Überzeugungen getragene Politiker wie Rómulo Betancourt, der langjährige Führer der AD und die COPEI-Leitfigur Rafael Caldera in der Zeit nach 1958 spielten.28 Auch externe Gegebenheiten spielten beim Erfolg der venezolanischen Tradition eine Rolle. Die Demokraten Venezuelas konnten sich jederzeit der Unterstützung der USA sicher sein.29 Die kubanische Einmischung und Förderung der marxistischen Guerilla bewirkte eine scharfe Abgrenzung der sozialdemokratischen $\mathrm{AD}$ gegenüber linkssozialistischen und antiparlamentarischen Kräften.

Gegenüber diesen herkömmlicherweise in den Mittelpunkt gerückten, außenpolitischen und externen Faktoren soll hier versucht werden, Variablen im politischen System selbst und deren Einfluß auf die Ergebnisse zu untersuchen. Ein politischer Erfolg, der gleichzeitig gerade kein ökonomischer und sozialer ist, kann sinnvoll nur mit politischen Variablen erklärt werden. Als zentrale Variablen werden untersucht Parteiensystem, Präsidentialismus/Parlamentarismus und Wahlsystem, wobei den Wechselwirkungen zwischen diesen Faktoren besondere Bedeutung zukommt. 30

\section{Das Parteiensystem}

Verhältnismäßig starke Beachtung fand in der wissenschaftlichen Literatur das venezolanische Parteiensystem 31 - zu Recht, sind doch die venezolanischen Parteien dominanter Träger des demokratischen Systems. Bemerkenswert und untypisch im lateinamerikanischen Vergleich ist dabei, daß sich hier ein kompetitives Parteiensystem mit einer Dominanz von zwei großen reformistischen Programmparteien herausgebildet hat. Traditionelle Statusparteien (Liberale/Konservative) sowie populistische oder autoritär-rechtsgerichtete Parteien konnten auf Dauer in Venezuela keine Bedeutung halten. Die Linke des Parteien-

28 Martz, John D./Myers, David J.: Venezuelan Democracy and the Future, in Martz/Myers (Hg.), a.a.O. (Fn.6), S.359-391, S.386.

29 Zur Außenpolitik Venezuelas umfassend Serbin, Andrés (Hg.), Venezuela y las relaciones internacionales en la Cuenca del Caribe, Caracas 1987.

30 so Nohlen, Dieter, a.a.O. (Fn.2).

31 Aus der zahlreichen Literatur siehe insbesondere die Arbeit von Werz, a.a.O. (Fn.9), passim.; Blank, Eugene E., Politics in Venezuela, Boston 1973; S.125-183; Martz, John D.: The Party System - Toward Institutionalization, in Martz/Myers (Hg.), a.a.O. (Fn.6), S.93-111; Manigat, Leslie: Venezuela, in Gott, Richard (Hrsg.). Guide to the Political Parties of Latin America, Harmondsworth 1973; ders. in Bernard, Jean Pierre (Hg.): Tableau des Partis Politiques en Amérique du Sud. Paris 1969. 
spektrums war lange Zeit von starker Zersplitterung und Fraktionismus gekennzeichnet. Die strukturell stärkste Partei Alianza Democratica hat sich im Laufe ihrer Geschichte von ihren traditionellen, populistischen Wurzeln gelöst und ist unter zeitweiliger Betonung liberal-demokratischer Vorstellungen zu einer demokratisch-reformistischen sozialdemokratischen Gruppierung geworden. Die inzwischen zweitstärkste Partei COPEI nahm demgegenüber stets eine christdemokratische Programmatik ein, oft stark reformistisch orientiert, die sie zweitweilig eher links der AD angesiedelt erscheinen ließ. Anderen Parteien, die es zahlreich gibt, kommt im nationalen Kräftespektrum keine große Bedeutung zu; die zu Beginn der Redemokratisierung zweitstärkste Partei URD, ist trotz ihrer Beteiligung am Punto-Fijo-Abkommen zwischenzeitlich völlig verschwunden. In Zukunft als dritte Kraft ernstzunehmen ist dagegen das 1988 erstmals vereint angetretene Linksbündnis MAS-MIR, das 10,27 Prozent der Stimmen erreichte. 1983 waren nur 7,32 Prozent auf die beiden Listen entfallen. Für eine Überraschung sorgte 1988 auch die rechts der Mitte stehende Gruppe "Nueva Generación" des Ex-COPEI-Abgeordneten Vladimir Gessen, die auf Anhieb mit 3,3 Prozent der Stimmen neun Sitze erreichte.

In der Struktur ihrer Wählerschaft zeichnen sich die venezolanischen Großparteien durch ein vergleichsweise hohes $\mathrm{Ma}$ an Homogenität aus. Während $\mathrm{AD}$ ursprünglich vor allem eine Partei der ländlichen Unterschichten war und COPEI vorwiegend in den westlichen Andenstaaten des Landes vertreten war, lassen sich inzwischen Stadt-/Land-, regionale oder soziale Cleavages kaum noch am Dualismus AD/COPEI festmachen.

\section{Der venezolanische Präsidentialismus}

Die lateinamerikanische Tradition präsidentieller Regierung - lange Zeit nahezu unangefochten - wird derzeit verstärkt kritischen Betrachtungen unterzogen. Bei einem Kolloquium zu diesem Thema sprachen sich nahezu alle anwesenden Politikwissenschaftlicher für eine Parlamentarisierung aus. 32 Dort war der venezolanische Präsidentialismus - und sinnvoll kann nur jedes Land bzw. sein Präsidentialismus für sich betrachtet werden - allerdings nicht Gegenstand der Debatte.

Präsidentialismus wirke kontraproduktiv, so die Argumentation der politikwissenschaftlichen Forschung33, weil er insbesondere im lateinamerikanischen Kontext negative Aspekte der lateinamerikanischen politischen Kultur34 - Caudillismus und Kazikentum, compra-

32 Nohlen/Solari(Hg.), a.a.O. (Fn.2).

33 Die wesentlichen Argumente der späteren Präsidentialismus-Kritik finden sich bereits bei Linz, Juan J., in Linz, Juan J./Stepan, A. (Hg.), The Breakdown of Democratic Regimes, Baltimore/ London 1978, Bd.1, S.71-74.

34 vgl. dazu ausführlich Mols, a.a.O. (Fn.4), S.51-131. 
drazgo $^{35}$ und Klientelismus36 - begünstige. Er lege den politischen Prozeß auf ein Nullsummenspiel des "Alles oder Nichts" fest, statt politische Absprachen, Kompromisse und Aufeinander-Zugehen zu begünstigen und mache den politischen Prozeß so zum "juego imposible". 37

Um Verfestigungen der Macht vorzubeugen, gilt auch in Venezuela ein Wiederwahlverbot für amtierende Präsidenten. Der Präsident ist - auch insofern bestehen in Venezuela keine Besonderheiten - gleichzeitig Staatsoberhaupt und Chef der Exekutive. Zusätzlich hat der venezolanische Präsident in der Praxis recht weitgehende legislative Befugnisse.

Kennzeichnend für die venezolanische Geschichte ist, daß die Präsidenten im allgemeinen eine hohe persönliche Wertschätzung auch über die Grenzen ihrer Partei hinaus genossen. Von 1959 bis 1964 hatte der Vater der AD, Rómulo Betancourt, das Amt inne; ihm folgte in der Amtsperiode 1964 bis 1969 Raúl Leoni (ebenfalls AD). 1969 kam es zum Machtwechsel, als Rafael Caldera, Gründer der christdemokratischen COPEI, die Präsidentschaftswahlen knapp gewann. Mit dem Wechsel von Caldera zu Carlos Andrés Pérez (AD) 1974 deutete sich die venezolanische Tradition alternierender Regierungen bereits an. Trotz der äußerst günstigen äußeren Rahmenbedingungen (Ölpreise) gelang es auch Pérez nicht, seinem Amtsbonus auf seinen Parteifreund zu übertragen; vielmehr gewann Luís Herrera Campins (COPEI) die Präsidentschaftswahlen 1978. In der folgenden Amtsperiode 19841989 folgte Jaime Lusinchi (AD) auf den Christdemokraten Herrera Campins.

Auch wenn es verfehlt wäre, dem venezolanischen Präsidenten eine omnipotente Stellung zuzuschreiben, ist er doch wichtigster Machtfaktor im politischen System. Seine weitreichenden sachlichen Entscheidungsbefugnisse, seine weitgehenden Möglichkeiten im Bereich der Ressourcenallokation und sein personeller Zugriff auf die gesamte Exekutive 38 tragen dazu bei. Danach gefragt, wer die mächtigste Institution des Landes sei, antworten denn auch 61 Prozent der Venezolaner mit "Regierung" oder "Präsident". Dagegen halten nur 17 Prozent den Kongreß für die mächtigste Institution. Das Militär kommt auf nur 3 Prozent, die Kirche gar auf 1, die Unternehmerverbände FEDECAMARAS auf 9 und die politischen Parteien auf 4 Prozent. Im lateinamerikanischen Kontext dürften diese Ergebnisse ziemlich aus dem Rahmen fallen. Bemerkenswert ist vor dem Hintergrund des skizzierten Präsidentialismus/Parlamentarismus-Debatte auch, da $\beta$ in der erwähnten Umfrage 60 Prozent meinten, die Stellung des Präsidenten solle weiter gestärkt werden.

37 ein unmögliches bzw. unspielbares Spiel.

38 vgl. Kelley, R. Lynn: Venezuelan Constitutional Forms and Realities, in Martz/Myers (Hg.), a.a.O. (Fn.6), S.27-45, S.28f. 
Lediglich 6 Prozent sprachen sich dafür aus, dem Präsidenten weniger Macht zuzubilligen.39 Eine Verfassungsreform in Richtung Parlamentarisierung würde vermutlich auf wenig Gegenliebe bei der Bevölkerung stoßen.

In der Verfassungspraxis ist der venezolanische Präsidentialismus weiter dadurch gekennzeichnet, daß das Parlament - der Kongreß - nur eine schwache Stellung innehat. Von einem wirksamen Gegengewicht im Sinne von "countervailing power" kann kaum gesprochen werden. Dies ist zum einen verfassungsrechtlich vorgegeben, wurde aber auch dadurch verstärkt, daß im Parlament über längere Zeiträume keine "regierungsfähigen" Mehrheiten bestanden. Ganz im Gegensatz zur landläufigen Einschätzung Venezuelas als Zweitparteiensystem bestand auch in Venezuela durchaus eine Tendenz zur Fragmentierung des Parteiensystems.

Die Folge dieser Entwicklung ist, daß selbst in die Domäne des Parlaments, die Gesetzgebung, der Präsident sehr weitgehend involviert ist. Ein sehr hoher Anteil der materiellen Gesetze Venezuelas ergeht als gesetzesvertretende Verordnung. Nach der Einschätzung eines venezolanischen Verfassungsrechtlers 40 sind Gesetze, die vom Kongreß beschlossen werden, geradezu "außergewöhnlich". Auch wenn man kein Befürworter parlamentarischer Regierungsweise in Lateinamerika ist, wird man diese mangelnde "capacidad legislativa" der Volksvertretung unter dem Aspekt des Demokratieprinzips und der Gewaltenteilung für bedenklich betrachten.

\section{Die Präsidentschaftswahlen}

Der venezolanische Präsident wird in einem Wahlgang in relativer Mehrheitswahl gewählt. Die Wahlen finden gleichzeitig mit den Parlamentswahlen statt. Bei den ersten Wahlen 1958 erreichte der Kandidat der AD, Rómulo Betancourt, dessen Name untrennbar mit dem Erfolg der venezolanischen Demokratie verbunden ist, unangefochtene 49,18 Prozent der abgegebenen "tar jeta grande"41 - Stimmen. Das zweitbeste Ergebnis erzielte COPEI-Kandidat Rafael Caldera mit nur 15,2 Prozent. 1963 schaffte es die AD trotz sich abzeichnender Spaltungstendenzen erneut, in Raul Leoni den Präsidenten zu stellen.

Der erste Machtwechsel 1968/69, der die venezolanische Tradition alternierender Regierungen begründete, wurde nur durch AD-Spaltungen ermöglicht. Dadurch konnte COPEI-

39 siehe Werz, a.a.O. (Fn.9), S.135.

40 Brewer-Carias, Allan: Instituciones políticas y constitucionales. 2. Auflage Caracas/Sán Cristóbal 1985, S.447.

41 Mit "tarjeta grande" ("große Karte") bezeichnen Venezolaner die Stimmzettel für die Präsidentschaftswahlen. Bei den gleichzeitig stattfindenden Parlamentswahlen wird dagegen mit einer "tarjeta pequeña" ("kleine Karte") abgestimmt. 
Kandidat Rafael Caldera mit 28,7 Prozent der Stimmen, ein für die zweitstärkste Partei damals sehr gutes Ergebnis, gewinnen. Der "offizielle" AD-Kandidat Barrios erreichte mit nur 25,5 Prozent ein Ergebnis, das weit unter dem Potential der lange Zeit hegemonischen Partei AD lag. Dies deshalb, weil die sich auf AD-Potential stützenden Kandidaten Burelli Rivas und Prieto 22,2 und 19,3 Prozent erzielten. Der die AD plagende Fraktionismus und die persönlichen, durchaus caudillistische Züge annehmenden Auseinandersetzungen wurden vom präsidentialistischen Wahlsystem erbarmungslos mit dem Machtverlust bestraft. Bei den Parlamentswahlen 1968 erreichte die offizielle AD, trotz ihrer sich auch dort auswirkenden Spaltung, immer noch eine - wenn auch knappe - relative Mehrheit. Die vielgelobte venezolanische Tradition altemierender Regierungen wäre aller Wahrscheinlichkeit in einem parlamentarischen System nicht zustande gekommen.

1973 kam nach der Überwindung ihrer Spaltungen emeut die AD an die Macht; auch die Christdemokraten konnten aber ihre Stellung ausbauen und immerhin 5 Prozent zulegen. Der Trend zum Zweiparteiensystem setzte sich 1978 fort, als COPEI-Kandidat Luís Herrera Campins mit 45,24 Prozent sich gegen seinen AD-Widersacher durchsetzte, der mit 43,3 Prozent ebenfalls gegenüber seinem Vorgänger zulegte. Auch 1978 erreichte die COPEI keine relative Mandatsmehrheit, obwohl sie erstmals auch bei den Parlamentswahlen mehr Stimmen erhielt als die AD.

Bei den Präsidentschaftswahlen 1983 konnte die AD ihre bereits verloren geglaubte Vorherrschaft wiederherstellen. Mit Jaime Lusinchi erreichte erstmals ein Bewerber eine klare absolute Mehrheit (56,85 Prozent), und dies obwohl die COPEI in dem Expräsidenten Rafael Caldera einen starken Gegenkandidaten aufgestellt hatte, der allerdings nur 34,56 Prozent der abgegebenen Stimmen erreichte. 1988 gelang Carlos Andres Pérez (AD) mit 52,9 Prozent die Rückkehr in den Präsidentenpalast. Sein Mitbewerber Eduardo Femández (COPEI), geschwächt durch die Rivalität mit Rafael Caldera, konnte das Ergebnis seiner Partei bei den Präsidentschaftswahlen gegenüber 1983 erheblich verbessem. Eine vernichtende Niederlage, wie sie von zahlreichen Beobachtern vorausgesagt worden war, blieb dem Christdemokraten erspart. Im Gegenteil ist er in einer recht guten Ausgangsposition für die nächsten Wahlen, nachdem er mit 40,2 Prozent der Stimmen das zweitbeste Ergebnis erreichte, das ein COPEI-Kandidat je erhalten hat.

Das System der relativen Mehrheitswahl ist in Venezuela dafür kritisiert worden, daß es bis 1983 stets dazu führte, daß Präsidenten mit einer "Minderheit", also weniger als 50 Prozent der Stimmen gewählt wurden. 42 Zur Verstärkung der demokratischen Legitimation wurde daher vorgeschlagen, auf ein System der absoluten Mehrheitswahl mit zwei Wahlgängen

42 vgl. Tovar, Orlando: Las instituciones electorales en Venezuela, in: Fundación Friedrich Ebert (Hg.): Sistemas Electorales y representación política en Latinoamerica, Madrid 1986, S.153. 
zurückzugreifen. An den historischen Tatsachen geht diese Argumentation vorbei: Eine absolute Mehrheitswahl hätte - wegen des Zwanges zu Wahlbündnissen - die Bedeutung kleiner Parteien gestärkt. Während das bestehende Wahlsystem der Präsidentschaftswahlen mit seinen praktischen und edukatorischen Auswirkungen dazu führte, daß die Stimmenbasis des Amtsinhabers von Mal zu Mal größer geworden ist, würde das angeblich "demokratischere" dies über einen zweiten Wahlgang nur herbeizwingen - ein Beispiel dafür, wie Wahlsystemkomponenten in der politischen Praxis oft gegenteilige Auswirkungen haben, wie ihnen prima facie zugeschrieben werden.

\section{Das venezolanische Wahlsystem (Parlamentswahlen)}

Vor dem Hintergrund der Dominanz des Präsidenten findet das venezolanische Wahlsystem in der politikwissenschaftlichen Literatur nur geringe Beachtung. 43 Vor dem Hintergrund der Vorschläge, Macht von der Institution Präsident auf das Parlament zu verlagern, ist dies nicht mehr gerechtfertigt. Das venezolanische Parlament besteht aus zwei Kammern, einem Senat und einem Abgeordnetenhaus. Neben den zum Urgestein okzidentaler Verfassungstradition zählenden allgemeinen Wahlrechtsgrundsätzen der freien, gleichen, geheimen und direkten Wahl sind die venezolanischen Parlamentswahlen durch eine ganze Reihe interessanter wahltechnischer Details gekennzeichnet.

Sowohl als Entscheidungsregel wie als Repräsentationsprinzip44 entschied sich die venezolanische Verfassung für die Verhältniswahl. Die Auszählung erfolgt nach d'Hondt auf Wahlkreisebene, die Stimmgebung im System starrer Listen. Die Wahlkreiseinteilung entspricht der föderalen Gliederung Venezuelas in 20 Bundesstaaten, die Hauptstadt Caracas und zwei Territorien. Die durchschnittliche Wahlkreisgröße beträgt somit 10 Mandate (im Abgeordnetenhaus) und ist hoch; dadurch kommt es bereits bei der Verteilung auf Wahlkreisebene zu einem hohen $\mathrm{Ma} \beta$ an Proportionalität. Gegenüber früher hat sich dies noch verstärkt, weil - bedingt durch die Bevölkerungsexplosion - die durchschnittliche Wahlkreisgröße gestiegen ist. Bis 1979 war nämlich die Größe des Parlaments bevölkerungsabhängig; auf 50.000 Einwohner entfiel ein Abgeordneter. Erst mit dem Wahlgesetz von 1977, das 1979 zum ersten Mal angewandt wurde, gibt es eine feste Zahl von Grundmandaten in Höhe von 1.982, d.h., auf 0,55 Prozent der Bevölkerung entfällt ein Sitz. Hinzu kommen Ausgleichsmandate.

43 siehe aber jüngst Martelo, Mercedes, El Sistema Electoral Venezolano. Resultados de la Aplicación del sistema de representacion proporcional en la composicion de las camaras del congreso, in Sintesis 5: Venezuela/Madrid 1988, S.221-252.

44 zu dieser Unterscheidung siehe Nohlen, Dieter: Wahlrecht und Parteiensystem, Opladen 1985, S.83ff. 
Obwohl bereits bei der Verteilung der Mandate auf Wahlkreisebene ein hohes Maß an Repräsentativität erreicht wird, sieht das venezolanische Wahlrecht zusätzlich Ausgleichsmandate für kleine Parteien vor. Nach der Verfassung darf das Quorum dafür maximal 1 Prozent betragen 45; das entsprechende Wahlgesetz legte die Quote für das Abgeordnetenhaus auf 0,55 Prozent fest.46 Jede Partei, die mehr als 5,5 Promille der abgegebenen Stimmen landesweit erreicht hat, ist damit im Kongreß vertreten. So verwundert es denn auch kaum, daß trotz der vielzitierten Tendenz zum Zweiparteiensystem (seit 1968 konnten die beiden großen Parteien AD und COPEI ihren gemeinsamen Stimmenanteil kontinuierlich von 49,5 auf 86,5 Prozent ausbauen) ein ausgesprochen zersplittertes Vielparteiensystem erhalten geblieben ist.

Zwar ist die Zahl der Ausgleichsmandate pro Partei beschränkt: Jede Gruppierung erhält (seit 1977) maximal 4 Abgeordnete bzw. 2 Senatoren. 1959-1977 betrugen die Zahlen 6 bzw. 4, 1958 lagen sie wie auch jetzt wieder bei 4 und 2. Die zahlreichen Splitterparteien sind dadurch aber kaum benachteiligt. Allenfalls die größeren unter den kleinen Parteien fallen unter diese "Kappungsgrenze". Sie erreichen jedoch in der Regel auch genügend Direktmandate in den großen Wahlkreisen. Ergebnis ist, daß die Kappungsgrenze so gut wie nie wirksam wurde und nur in außergewöhnlichen Konstellationen jemals wirksam werden kann.

Die Zahl der im Parlament vertretenen Parteien betrug im gleichen Zeitraum stets unverändert elf. Daß es völlig entgegen den Tendenzen bei den Parlamentswahlen zu einem Zweiparteiensystem kam, ist alleine auf die Variable Präsidentialismus zurückzuführen. Das Parlamentswahlsystem dagegen wirkt nicht in diese Richtung. 47

Die Wahlen am 4. Dezember 1988 bestätigten diese Hypothese emeut. Auf die Präsidentschaftskandidaten der Großparteien AD und COPEI entfielen 93,1 Prozent. Dies ist mehr als jemals zuvor. Lediglich der Kandidat des Linksbündnisses MAS-MIR, Teodoro Petkoff, konnte 2,7 Prozent erreichen.

Bei den Parlamentswahlen dagegen konnten die beiden Großparteien nur 75,19 Prozent erreichen. Hier ist die Konzentration damit erstmals wieder spürbar zurückgegangen. Nach jahrelangen Konzentrationstendenzen wurden hier zentrifugale Tendenzen sichtbar.

45 Art. 151 der Verfassung von 1961.

46 Art. 3 de Ley Orgánica del Sufragio von 1977. Technisch wird dies erreicht, indem die Gesamtstimmenzahl durch die Gesamt-Sitzzahl (jetzt mit $182 \mathrm{fix}$ ) geteilt wird. Vorher hatte die Quote langfristig abgenommen, weil mit steigender Bevölkerung die Sitzzahl stieg (1958 126 Sitze, 1963 168 Sitze, 1968197 Sitze, 1973183 Sitze). Daraus errechnen sich die Quoten 0,761, 0,595, 0,50 und 0,5465 .

47 so auch Rey, Juán Carlos: El futuro de la democracia en Venezuela, in Silva Michalena, José A. (Hg.), Venezuela hacía e12000, Caracas 1987, S.183-245, S.235. 
Die konkreten Wirkungen des Präsidentialismus, die diesen Effekt herbeiführten, sind mannigfaltig: Der Präsidentialismus bestrafte Spaltungstendenzen in den beiden Großparteien und verhinderte so eine weitere Fragmentierung. Da die Pfründe einzig von der Exekutive verteilt werden und bei den Präsidentschaftswahlen nur die beiden Großparteien eine Chance haben, werden kleine Parteien - trotz des leichten Parlamentszuganges - unattraktiv. $\mathrm{Daß}$ die Wahlkämpfe stark auf die beiden Großparteien zugeschnitten sind, liegt ebenfalls daran, daß bei den für wichtiger erachteten Präsidentschaftswahlen kleine Parteien (ganz im Gegensatz zu den Parlamentswahlen) keine Chance haben. Der oft hervorgehobene Grundsatz der Wahlökonomie, der Venezolaner angeblich von einer Stimmabgabe für die kleineren Parteien abhält, ist ebenfalls einzig auf die gleichzeitig stattfindenden Präsidentschaftswahlen zurückzuführen. Bei den Parlamentswahlen gibt es Dank der äußerst niedrigen Zugangsbarriere kaum verschenkte Stimmen. Auch anhand der Zahlen zeigt sich die durch den Präsidentialismus katalysierte Tendenz zum Zweiparteiensystem: In beiden Wahlen Parlament und Präsident - stieg der gemeinsame Anteil der beiden großen Parteien seit 1968 kontinuierlich. Dabei folgte der gemeinsame AD/COPEI-Anteil dem gemeinsamen Anteil der AD/COPEI-Bewerber zeitverzögert. Bei der letzten Wahl 1988 ging der kombinierte Anteil bei den Parlamentswahlen nun deutlich zurück, bei den Präsidentschaftswahlen dagegen stieg er weiter an und liegt nun bei weit über 90 Prozent.

\section{Die konkreten Auswirkungen des Präsidentialismus in Venezuela}

Als Ergebnis läßt sich festhalten, daß der Präsidentialismus die demokratietheoretisch und praktisch allgemein gutgeheißene Praxis alternierender Regierungen und die Herausbildung eines demokratietragenden Zweiparteiensystems erst ermöglichte. Nur bei den Präsidentschaftswahlen konnte die strukturell zweitstärkste Partei COPEI die Mehrheit erreichen. Die starke Ausrichtung der venezolanischen Wahlkämpfe auf die Präsidentschaftswahlen bietet gegenüber reinen Parteienwahlen eine Legitimationswirkung für das politische System, indem den Wählem vereinfachte Identifikationsmöglichkeiten, eine stärkere Reduktion der Komplexität schon im Vorfeld der Wahlen ermöglicht werden. Gegen die Kritik, der Präsidentialismus leiste caudillistischen und personalistischen Tendenzen Vorschub, läßt sich durchaus die Gegenthese aufstellen, wonach der venezolanische Präsidentialismus zur Einigkeit zwingt und gezwungen hat, personell bedingten Spaltungen entgegenwirkt, weil er derartige Entwicklungen pönalisiert. Weiter trug er entscheidend dazu bei, das venezolanische Zweiparteiensystem herauszubilden; nur bei den Präsidentschaftswahlen kommt es zu einer Ausrichtung des Wählerverhaltens am Grundsatz der Wahlökonomie, wonach Stimmen, die nicht für AD oder COPEI abgegeben werden, als verloren gelten. Die Komponente Präsidentialismus schafft so ein unerläßliches Gegengewicht zum stark fragmentierenden Parlaments-Wahlsystem. Daß Venezuela rotz oft fehlender Parlamentsmehrheiten stets regierungsfähig blieb, ist ebenfalls auf den Präsiden- 
tialismus zurückzuführen. Das Beispiel Venezuela zeigt, daß eine allgemeine These, wonach parlamentarische Regierungen besser als präsidentielle für die Stabilisierung demokratisch-konstitutioneller Systeme geeignet sind, falsch ist. 


\section{ABSTRACTS}

\section{Brazil: Politics and Catholic Church in the "New Republic"}

\section{By Christiano German}

The paper discusses three fields of conflict between government and Catholic Church in Brazil's "New Republic" from 1985 to 1989 . The analysis of the confrontations about the role of the National Security Council, the Indian question and the problems of mass communication shows a marked dissent as to the view of the speed and the quality of the democratization process. Whereas the attitude of the govemment was rather passive and socio-conservative, the Catholic Church took an active, progress-oriented position. The decisions of the Constituent Assembly, which played a special role during this period, were included into the analysis of the respective fields of conflict. It was found that concerning the issues under examination the "Constituintes" - inspite of massive attempts by opponents of a new order to exercise political influence - were able to put through consent- and reform-oriented constitutional laws. The respective articles of Brazil's new 1988 constitution point, for the time being only theoretically, at a kind of middle way between the positions of the government and the Catholic Church, the arguments presented by the Church tending to show more power of persuasion.

\section{Democracy in Venezuela: Presidentialism, Political Parties and Elections}

\section{By Wolfgang Spoerr}

The author concludes that the fact that the Venezuelan democracy is presidential and not parliamentary has contributed decisively to the democratic stability of this country. The competing hypotheses of "political pacts" is rejected as having little explanatory value. Without presidential elections, it is likely that the bipartisan party system in Venezuela would not have evolved. The electoral system of parliamentary elections is characterized as being highly proportionate. Elements which were designed to reduce the influence of small parties have not become effective in Parliamentary elections. Instead, the presidential access to the executive branch of government has served the purpose of focusing political interest and activities in the two big parties $\mathrm{AD}$ and COPEI. 